\title{
Near Optimal Work-Stealing Tree Scheduler for Highly Irregular Data-Parallel Workloads
}

\author{
Aleksandar Prokopec, Martin Odersky \\ École Polytechnique Fédérale de Lausanne, Lausanne, Switzerland
}

\begin{abstract}
We present a work-stealing algorithm for runtime scheduling of dataparallel operations in the context of shared-memory architectures on data sets with highly-irregular workloads that are not known a priori to the scheduler. This scheduler can parallelize loops and operations expressible with a parallel reduce or a parallel scan. The scheduler is based on the work-stealing tree data structure, which allows workers to decide on the work division in a lock-free, workloaddriven manner and attempts to minimize the amount of communication between them. A significant effort is given to showing that the algorithm has the least possible amount of overhead.

We provide an extensive experimental evaluation, comparing the advantages and shortcomings of different data-parallel schedulers in order to combine their strengths. We show specific workload distribution patterns appearing in practice for which different schedulers yield suboptimal speedup, explaining their drawbacks and demonstrating how the work-stealing tree scheduler overcomes them. We thus justify our design decisions experimentally, but also provide a theoretical background for our claims.
\end{abstract}

\section{Introduction}

In data-parallel programming models parallelism is not expressed as a set process interactions but as a sequence of parallel operations on data sets. Programs are typically composed from high-level data-parallel operations, and are declarative rather than imperative in nature, which is of particular interest when it comes to programming the ever more present multicore systems. Solutions to many computational problems contain elements which can be expressed in terms of data-parallel operations [12].

We show several examples of data-parallel programs in Figure 1. These programs rely heavily on higher-order data-parallel operations such as map, reduce and filter, which take a function argument - they are parametrized by a mapping function, a reduction operator or a filtering predicate, respectively. The first example in Figure 1 computes the variance of a set of measurements ms. It starts by computing the mean value using the higher-order operation sum, and then maps each element of ms into a set of squared distances from the mean value, the sum of which divided by the number of elements is the variance $v$. The amount of work executed for each measurement value is equal, so we call this workload uniform. This need not be always so. The second program computes all the prime numbers from 3 until $N$ by calling a data-parallel filter on the corresponding range. The filter uses a predicate that checks that no number from 2 to $\sqrt{i}$ divides $i$. The workload is not uniform nor independent of $i$ and 
the processors working on the end of the range need to do more work. This example also demonstrates that data-parallelism can be nested - the forall can be done in parallel as each element may require a lot of work. On the other hand, the reduce in the third program that computes a sum of numbers from 0 to $N$ requires a minimum amount of work for each element. A good data-parallel scheduler must be efficient for all the workloads - when executed with a single processor the reduce in the third program must have the same running time as the while loop in the fourth program, the data-parallelism of which is not immediately obvious due to its imperative style.

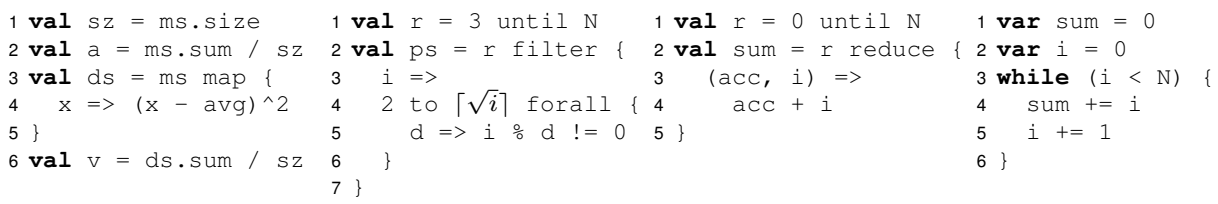

Fig. 1. Data parallel program examples

It has been a trend in many languages to provide data-parallel bulk operations on collections [3] [4] [5] [17] [18]. Data-parallel operations are generic as shown in Figure 1 - for example, reduce takes a user-provided operator, such as number addition, string concatenation or matrix multiplication. The computational costs of these generic parts, and hence the workload distribution, cannot always be determined statically, so efficient assignment of work to processors often relies on the runtime scheduling. Scheduling in this case entails dividing the elements into batches on which the processors work in isolation. Work-stealing [1] [8] [7] [15] [20] is one solution to this problem. In this technique different processors occasionally steal batches from each other to load balance the work - the goal is that no processor stays idle for too long.

In this paper we propose and describe a runtime scheduler for data-parallel operations on shared-memory architectures that uses a variant of work-stealing to ensure proper load-balancing. The scheduler relies on a novel data structure with lock-free synchronization operations called the work-stealing tree. To show that the work-stealing tree scheduler is optimal we focus on evaluating scheduler performance on uniform workloads with a minimum amount of computation per element, irregular workloads for which this amount varies and workloads with a very coarse granularity.

Our algorithm is based on the following assumptions. There are no fast, accurate means to measure elapsed time with sub-microsecond precision, i.e. there is no way to measure the running time. There is no static or runtime information about the cost of an operation - when invoking a data-parallel operation we do not know how much computation each element requires. There are no hardware-level interrupt handling mechanisms at our disposal - the only way to interrupt a computation is to have the processor check a condition. We assume OS threads as parallelism primitives, with no control over the scheduler. We assume that the available synchronization primitives are monitors and the CAS instruction. We assume the presence of automatic memory management.

The rest of the paper is organized as follows. Section 2 describes related work and alternative schedulers we compare against. Section 3 describes the work-stealing tree scheduler. In Section 4 we evaluate the scheduler for different workloads as well as tune several of its parameters, and in Section 5 we conclude. 


\section{Related work}

Per processor (henceforth, worker) work assignment done statically during compile time or linking, to which we will refer to as static batching, was studied extensively [13] [19]. Static batching cannot correctly predict workload distributions for any problem, as shown by the second program in Figure 1. Without knowing the numbers in the set exactly, batches cannot be statically assigned to workers in an optimal way - some workers may end up with more work than the others. Still, although cost analysis is not the focus here, we advocate combining static analysis with runtime techniques.

To address the need for load balancing at runtime, work can be divided into a lot of small batches. Only once each worker processes its batch, it requests a new batch from a centralized queue. We will refer to this as fixed-size batching [14]. In fixedsize batching the workload itself dictates the way how work is assigned to workers. This is a major difference with respect to static batching. In general, in the absence of information about the workload distribution, scheduling should be workload-driven. A natural question arises - what is the ideal size for a batch? Ideally, a batch should consist of a single element, but the cost of requesting work from a centralized queue is prohibitively large for that. For example, replacing the increment $i+=1$ with an atomic CAS can increase the running time of a while loop by nearly a magnitude on modern architectures. The batch size has to be the least number of elements for which the cost of accessing the queue is amortized by the actual work. There are two issues with this technique. First, it is not scalable - as the number of workers increases, so does contention on the work queue (Figure 6). This requires increasing batch sizes further. Second, as the granularity approaches the batch size, the work division is not fine-grained and the speedup is suboptimal (Figure 8, where size is less than 1024).

Guided self-scheduling [16] solves some granularity issues by dynamically choosing the batch size based on the number of remaining elements. At any point, the batch size is $R_{i} / P$, where $R_{i}$ is the number of remaining elements and $P$ is the number of workers - the granularity becomes finer as there is less and less work. Note that the firstarriving worker is assigned the largest batch of work. If this batch contains more work than the rest of the loop due to irregularity, the speedup will not be linear. This is shown in Figures 8-20, 9-35. Factoring [10] and trapezoidal self-scheduling [21] improve on guided-self scheduling, but have the same issue with those workload distributions.

One way to overcome the contention issues inherent to the techniques above is to use several work queues rather than a centralized queue. In this approach each processor starts with some initial work on its queue and commonly steals from other queues when it runs out of work - this is known as work-stealing, a technique applicable to both taskand data-parallelism. One of the first uses of work-stealing dates to the Cilk language [2] [8], in which processors relied on the fast and slow version of the code to steal stack frames from each other. Recent developments in the X10 language are based on similar techniques [20]. Work-stealing typically relies on the use of work-stealing queues [1] [7] [8] [15] and deques [6], implementations ranging from blocking to lock-free. While in the past data-parallel collections frameworks relied on using task-parallel schedulers under the hood [11] [17] [18], to the best of our knowledge, the tree data structure was not used for synchronization in work-stealing prior to this work, nor for data-parallel operation scheduling. 
a $\mathrm{C}$ structure. We define two compound data-types. Ptr is a reference to the tree it has only a single member child of type Node. Write access to child has to be atomic and globally visible (in Java, this is ensured with the volatile keyword). Node contains immutable references to the left and right subtree, initialized upon instantiation. If these are set to $\perp$ we consider the node a leaf. We initially focus on parallelizing loops over ranges, so we encode the current state of iteration with three integers. Members start and until are immutable and denote the initial range for the root of the tree this is the entire loop range. Member progress has atomic, globally visible write access. It is initially set to start and is updated as elements are processed. Finally, the owner field denotes the worker that is working on the node. It is initially $\perp$ and also has atomic write access. Example trees are shown in Figure 3.

Before we describe the operations and the motivation behind these data-types we will define the states work-stealing tree can be in (see Figure 3), namely its invariants. This is of particular importance for concurrent data structures which have non-blocking operations. Work-stealing tree operations are lock-free, a well-known advantage [9], which comes at the cost of little extra complexity in this case.

INV1 Whenever a new node reference Ptr $\mathrm{p}$ becomes reachable in the tree, it initially points to a leaf Node $\mathrm{n}$, such that $\mathrm{n}$. owner $=\perp$. Field $\mathrm{n}$.progress is set to $\mathrm{n}$. start and $\mathrm{n}$. until $\geq \mathrm{n}$. start. The subtree is in the AVAILABLE state and its range is $\langle n$. start, n. until $\rangle$.

INV2 The set of transitions of $\mathrm{n}$. owner is $\perp \rightarrow \pi \neq \perp$. No other field of $\mathrm{n}$ can be written until $\mathrm{n}$. owner $\neq \perp$. After this happens, the subtree is in the OWNED state.

INV3 The set of transitions of n.progress in the OWNED state is $p_{0} \rightarrow p_{1} \rightarrow$ $\ldots \rightarrow p_{k}$ such that $\mathrm{n}$. start $=p_{0}<p_{1}<\ldots<p_{k}<\mathrm{n}$. until. If a worker $\pi$ writes a value from this set of transitions to $\mathrm{n}$. progress, then $\mathrm{n}$. owner $=\pi$.

INV4 If the worker $n$. owner writes the value $n$. until to $n$.progress, then that is the last transition of $\mathrm{n}$. progress. The subtree goes into the COMPLETED state.

INV5 If a worker $\psi$ overwrites $p_{i}$, such that $\mathrm{n}$. start $\leq p_{i}<\mathrm{n}$. until, with $p_{s}=$ $-p_{i}-1$, then $\psi \neq \mathrm{n}$. owner. This is the last transition of $\mathrm{n}$.progress and the subtree goes into the STOLEN state.

INV6 The field $p$. child can be overwritten only in the STOLEN state, in which case its transition is $\mathrm{n} \rightarrow \mathrm{m}$, where $\mathrm{m}$ is a copy of $\mathrm{n}$ with $\mathrm{m}$. left and $\mathrm{m}$. right being fresh leaves in the AVAILABLE state with ranges $r_{l}=\left\langle x_{0}, x_{1}\right\rangle$ and $r_{r}=\left\langle x_{1}, x_{2}\right\rangle$ such that $r_{l} \cup r_{r}=\left\langle p_{i}, \mathrm{n}\right.$. until $\rangle$. The subtree goes into the EXPANDED state.

This seemingly complicated set of invariants can be summarized in a straightforward way. Upon owning a leaf, that worker processes elements from that leaf's range by incrementing the progress field until either it processes all elements or another worker requests some work by invalidating progress, in which case the leaf is replaced by a subtree such that the remaining work is divided between the new leaves.

Now that we have formally defined a valid work-stealing tree, we provide an implementation of the basic operations (Figure 4). These operations will be the building blocks for the scheduling algorithm that balances the workload. A worker must attempt to acquire ownership of a node before processing its elements by calling the method tryOwn, which returns true if the claim is successful. After reading the owner field in line 14 and establishing the AVAILABLE state, the worker attempts to atomically 
Parallelizing the baseline seems trivial. Assuming the workers start at roughly the same time and have roughly the same speed, we can divide the range in equal parts between them. However, an assumption from the introduction was that the workload distribution is not known and the goal is to parallelize irregular workloads as well. In fact, the workload may have a coarse granularity, consisting only of several elements.

For the reasons above, we verify that the scheduler abides the following criteria:

C1 There is no noticeable overhead when executing the baseline with a single worker. C2 Speedup is optimal for both the baseline and typical irregular workloads.

C3 Speedup is optimal when the work granularity equals the parallelism level.

Workloads we choose correspond to those found in practice. Uniform workloads are particularly common and correspond to numeric computations, text manipulation, Monte Carlo methods and applications that involve basic linear algebra operations like vector addition or matrix multiplication. In Figure 8 we denote this workload as UNIFORM. Triangular workloads are present in primality testing, multiplication with triangular matrices and computing an adjoint convolution (TRIANGLE). In higher dimensions computing a convolution consists of several nested loops and can have a polynomial workload distribution (PARABOLA). Depending on how the problem is formulated, the workload may be increasing or decreasing (INVTRIANGLE, HILL, VALLEY). In combinatorial problems such as word segmentation, bin packing or computing anagrams the problem subdivision can be such that the subproblems corresponding to different elements differ exponentially - we model this with an exponentially increasing workload EXP. In raytracing, PageRank or sparse matrix multiplication the workload corresponds to some probability distribution, modelled with workloads GAUSSIAN and RANDIF. Finally, in problems like Mandelbrot set computation or Barnes-Hut simulation we have large conglomeration of elements which require a lot of computation while the rest require almost no work. We call this workload distribution STEP.

All the tests were performed on an Intel i7 $3.4 \mathrm{GHz}$ quad-core processor with hyperthreading and Oracle JDK 1.7, using the server VM. Our implementation is written in the Scala programming language, which uses the JVM as its backend. JVM programs are commonly regarded as less efficient than programs written in $\mathrm{C}$. To show that the evaluation is comparative to a $\mathrm{C}$ implementation, we must evaluate the performance of corresponding sequential $\mathrm{C}$ programs. The running time of the while loop from Figure 1 is roughly $45 \mathrm{~ms}$ for 150 million elements in both $\mathrm{C}$ (GNU C++ 4.2) and on the JVM - if we get linear speedups then we can conclude that the scheduler is indeed optimal. We can thus turn our attention to critera $\mathrm{C} 1$.

We stated already that the STEP value should ideally be 1 for load-balancing purposes, but has to be more coarse-grained due to communication costs that could overwhelm the baseline. In Figure 6A we plot the running time against the STEP size, obtained by executing the baseline loop with a single worker. By finding the minimum STEP value with no observable overhead, we seek to satisfy criteria $\mathrm{C} 1$. The minimum STEP with no noticeable synchronization costs is around 50 elements - decreasing STEP to 16 doubles the execution time and for value 1 the execution time is 36 times larger (not shown for readability).

Having shown that the work-stealing tree is as good as fixed-size batching, we evaluate its effectiveness with multiple workers. Figure 6B shows that the minimum STEP 

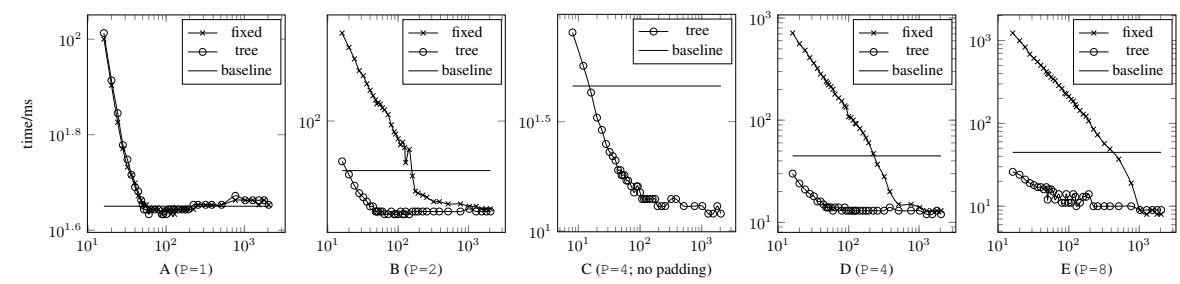

Fig. 6. Baseline running time (ms) vs. STEP size

for fixed-size batching increases for 2 workers, as we postulated earlier. Increasing STEP decreases the frequency of synchronization and the communication costs with it. In this case the $3 \mathrm{x}$ slowdown is caused by processors having to exchange ownership of the progress field cache-line. The work-stealing tree does not suffer from this problem, since it strives to keep processors isolated - the speedup is linear with 2 workers. However, with 4 processors the performance of the naive work-stealing tree implementation is degraded (Figure 6C). While the reason is not immediately apparent, note that for greater STEP values the speedup is once again linear. Inspecting the number of elements processed in each node reveals that the uniform workload is not evenly distributed among the topmost nodes - communication costs in those nodes are higher due to false sharing. Even though the two processors work on different nodes, they modify the same cache line, slowing down the CAS in line 20 . Why this exactly happens in the implementation that follows directly from the pseudocode is beyond the scope of this paper, but it suffices to say that padding the node object with dummy fields to adjust its size to the cache line solves this problem, as shown in Figures 6D,E.

The speedup is still not completely linear as the number of workers grows. Our baseline does not access main memory and only touches cache lines in exclusive mode, so this may be due to worker wakeup delay or scheduling costs in the work-stealing tree. After checking that increasing the total amount of work does not change performance, we focus on the latter. Inspecting the number of tree nodes created at different parallelism levels in Figure 7B reveals that as the number of workers grows, the number of nodes grows at a superlinear rate. Each node incurs a synchronization cost, so could we decrease their total number?

Examining a particular work-stealing tree instance at the end of the operation reveals that different workers are battling for work in the left subtree until all the elements are depleted, whereas the right subtree remains unowned during this time. As a result, the workers in any subtree steal from each other more often, hence creating more nodes. The cause is the left-to-right tree traversal in findWork as defined in Figure 5, a particularly bad stealing strategy we will call Predefined. As shown in Figure $7 \mathrm{~B}$, the average tree size for 8 workers nears 2500 nodes. So, lets try to change the preference of a worker by changing the tree-traversal order in line 70 based on the worker index $i$ and the level $l$ in the tree. The worker should go left-to-right if and only if $\left(i>>\left(l \bmod \left\lceil\log _{2} P\right\rceil\right)\right) \bmod 2=1$ where $P$ is the total number of workers. This way, the first path from the root to a leaf up to depth $\log _{2} P$ is unique for each worker. The choice of the subtree after a steal in lines 10 and 66 is also changed like this - the detailed implementation of findWork for this and other strategies is shown 
in the appendix. This strategy, which we call Assign, decreases the average tree size at $P=8$ to 134 . Interestingly, we can do even better by doing this assignment only if the node depth is below $\log _{2} P$ and randomizing the traversal order otherwise. We call this strategy AssignTop - it decreases the average tree size at $P=8$ to 77. Building on the randomization idea, we introduce an additional strategy called RandomWalk where the traversal order in findWork is completely randomized. However, this results in a lower throughput and bigger tree sizes. Additionally randomizing the choice in lines 10 and 66 (RandomAll) is even less helpful, since the stealer and the victim clash more often.
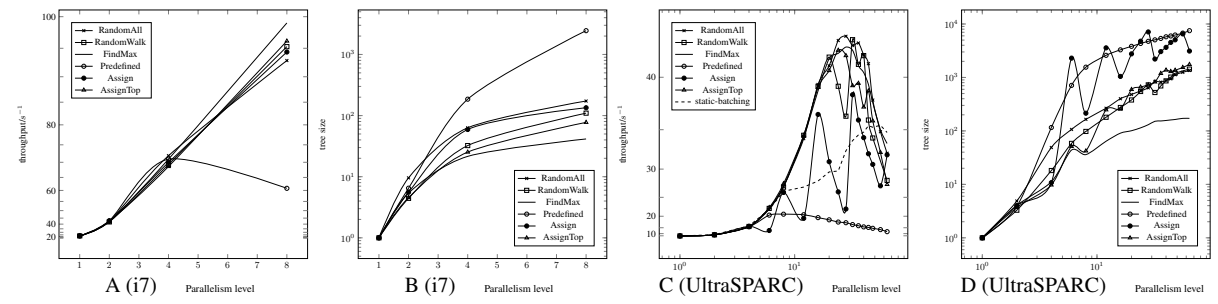

Fig. 7. Comparison of $\mathrm{findWork}$ implementations

The results of the five different strategies mentioned thus far lead to the following observation. If a randomized strategy like RandomWalk or AssignTop works better than a suboptimal strategy like Predefined then some of its random choices are beneficial to the overall execution time and some are disadvantageous. So, there must exist an even better strategy which only makes the choices that lead to a better execution time. Rather than providing a theoretical background for such a strategy, we propose a particular one which seems intuitive. Let workers traverse the entire tree and pick a node with most work, only then attempting to own or steal it. We call this strategy FindMax. Note that this cannot be easily implemented atomically, but a quiescently consistent implementation may still serve as a decent heuristic. This strategy yields an average tree size of 42 at $P=8$, as well as a slightly better throughput - we conclude by choosing it as our default strategy. Also, the diagrams in Figure 7 reveal the postulated inverse correlation between the tree size and total execution time, both for the Intel i7-2600 and the Sun UltraSPARC T2 processor (where STEP is set to 600), which is particularly noticeable for Assign when the total number of workers is not a power of two. For some $P$ RandomAll works slightly better than FindMax on UltraSPARC, but both are much more efficient than static batching, which deteriorates heavily once $P$ exceeds the number of cores.

The results so far go a long way in justifying that $\mathrm{C} 1$ is fulfilled. We focus on the $\mathrm{C} 2$ and $\mathrm{C} 3$ next by changing the workloads, namely the kernel function. Figures 8, 9 show a comparison of the work-stealing tree and the other schedulers on a range of different workloads. Each workload pattern is illustrated prior to its respective diagrams, along with corresponding real-world examples. To avoid memory access effects and additional layers of abstraction each workload is minimal and synthetic, but corresponds to a practical use-case. To test C3, in Figure 8-5,6 we decrease the number of elements to 16 and increase the workload heavily. Fixed-size batching fails utterly for these workloads - the total number of elements is on the order of or well below the 

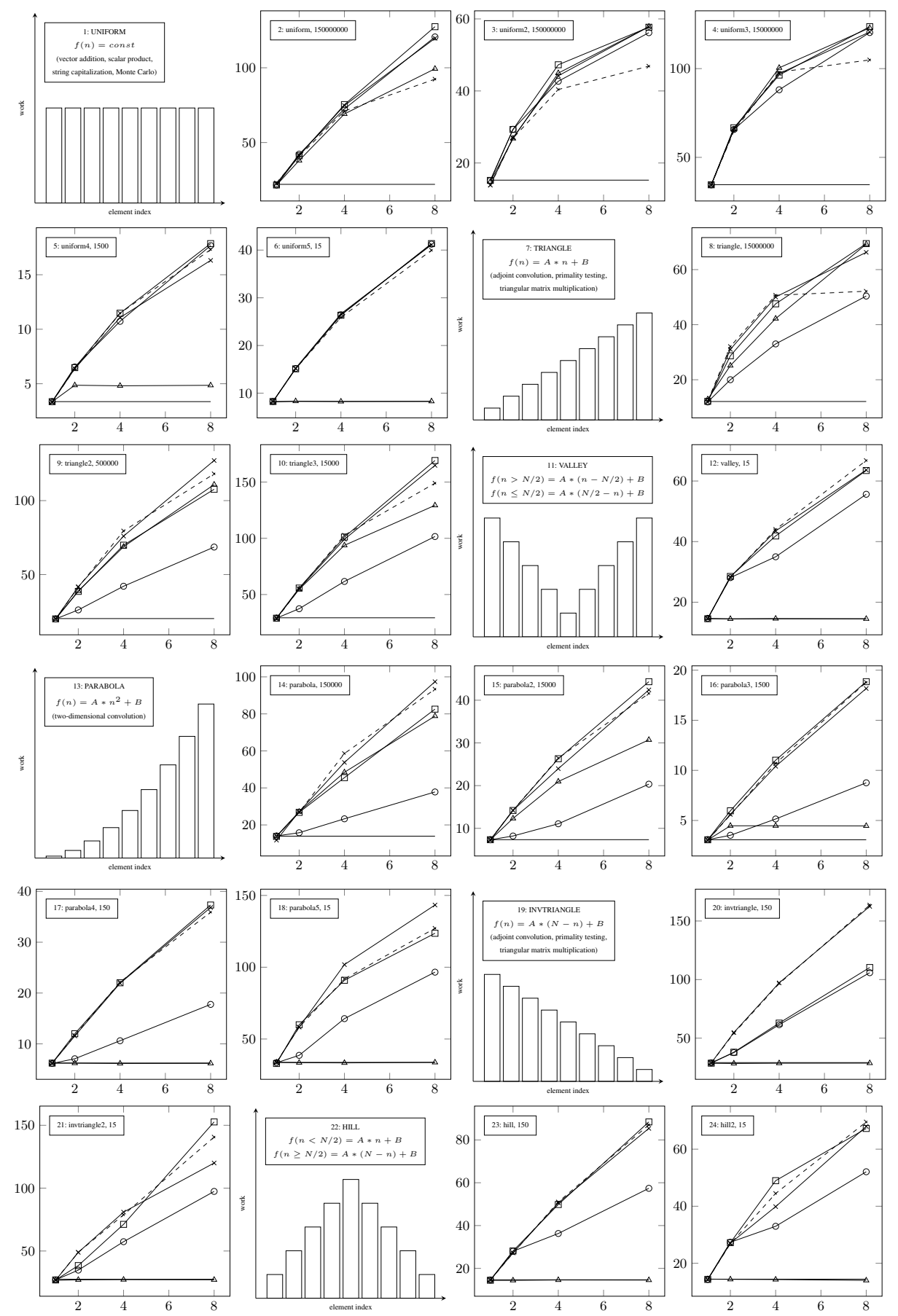

- baseline $\bigcirc-$ static-batching $\triangle$ fixed-size $\quad \square$ GSS $\quad \star$ tree-findmax $\quad-\star-$ tree-predef

Fig. 8. Comparison of different kernel functions I (throughput $/ s^{-1}$ vs. \#workers) 

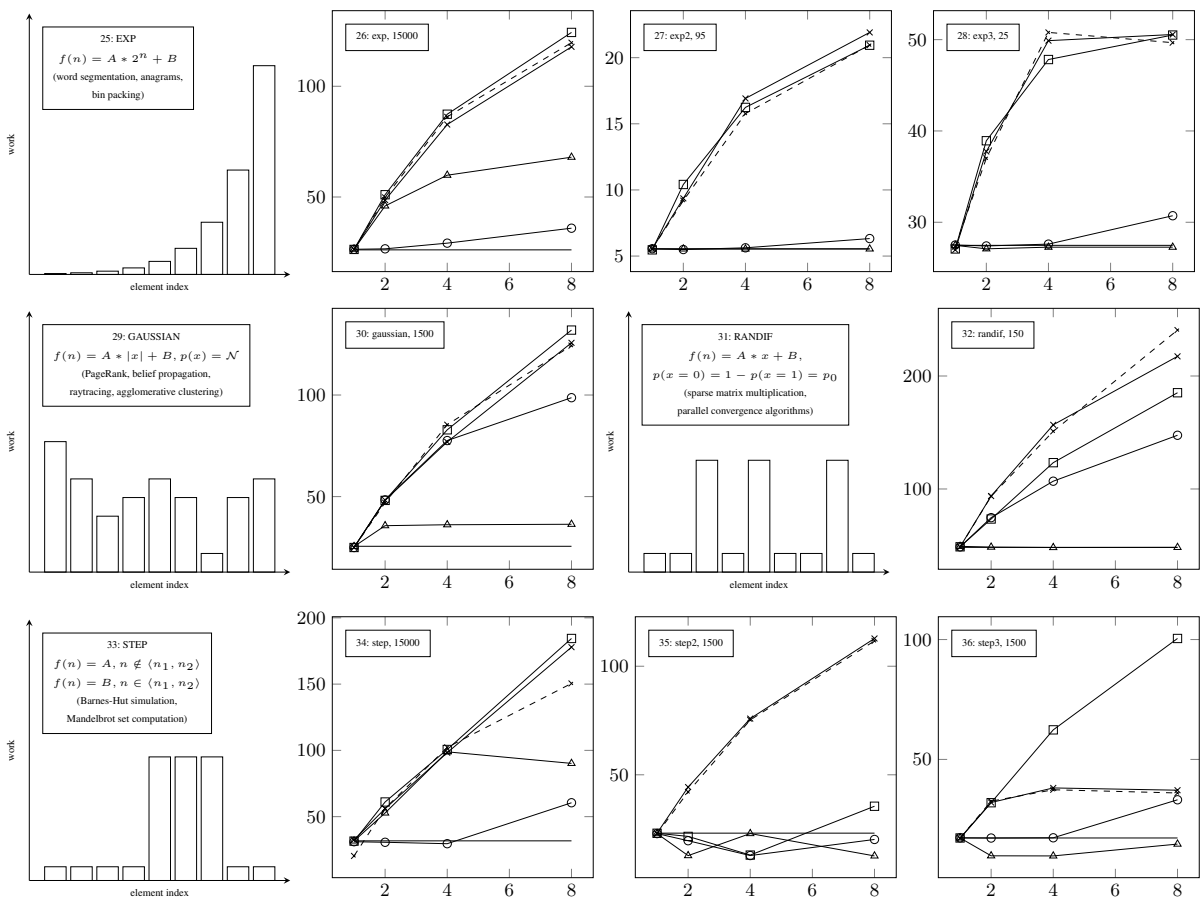

— baseline $\multimap$ static-batching $\triangle$ fixed-size $\boxminus$ GSS $\quad *$ tree-findmax $\quad-\star$ - tree-predef

Fig. 9. Comparison of different kernel functions II (throughput $/ s^{-1}$ vs. \#workers)

estimated STEP. These workloads obviously require smaller STEP sizes to allow stealing, but that would annul the baseline performance, and we cannot distinguish the two. We address these seemingly incompatible requirements by modifying the work-stealing tree in the following way. A mutable step field is added to Node, which is initially 1 and does not require atomic access. At the end of the while loop in the workOn method the step is doubled unless greater than some value MAXSTEP. As a result, workers start processing each node by cautiously checking if they can complete a bit of work without being stolen from and then increase the step exponentially. This naturally slows down the overall baseline execution, so we expect the MAXSTEP value to be greater than the previously established STEP. Indeed, on the i7-2600, we had to set MAXSTEP to 256 to maintain the baseline performance and at $P=8$ even 1024 . With these modifications work-stealing tree yields linear speedup for all uniform workloads.

Triangular workloads such as those shown in Figures 8-8,9,10 show that static batching can yield suboptimal speedup due to the uniform workload assumption. Figure 8-20 shows the inverse triangular workload and its negative effect on guided selfscheduling - the first-arriving processor takes the largest batch of work, which incidentally contains most work. We do not inverse the other increasing workloads, but stress that it is neither helpful nor necessary to have batches above a certain size. 

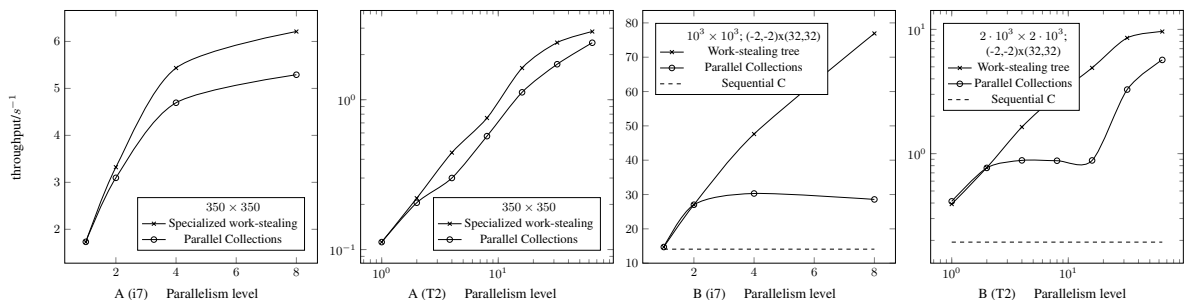

Fig. 10. (A) Matrix multiplication and (B) Mandelbrot sets on i7 and UltraSPARC T2

Figure 9-28 shows an exponentially increasing workload, where the work associated with the last element equals the rest of the work - the best possible speedup is 2. Figures 9-30,32 show two examples where a probability distribution dictates the workload, which occurs often in practice. Guided self-scheduling works well when the distribution is relatively uniform, but fails to achieve optimal speedup when only a few elements require more computation, for reasons mentioned earlier.

In the STEP distributions all elements except those in some range $\left\langle n_{1}, n_{2}\right\rangle$ are associated with a very low amount of work. The range is set to $25 \%$ of the total number of elements. When its absolute size is above MAXSTEP, as in Figure 9-34, most schedulers do equally well. However, not all schedulers achieve optimal speedup as we decrease the total number of elements $N$ and the range size goes below MAXSTEP. In Figure 9-35 we set $n_{1}=0$ and $n_{2}=0.25 N$. Schedulers other than the work-stealing tree achieve almost no speedup, each for the same reasons as before. However, in Figure 9-36, we set $n_{1}=0.75 N$ and $n_{2}=N$ and discover that the work-stealing tree achieves a suboptimal speedup. The reason is the exponential batch increase - the first worker acquires a root node and quickly processes the cheap elements, having increased the batch size to MAXSTEP by the time it reaches the expensive ones. The real work is thus claimed by the first worker and the others are unable to acquire it. Assuming some batches are smaller and some larger as already explained, this problem cannot be worked around by a different batching order - there always exists a workload distribution such that the expensive elements are in the largest batch. In this adversarial setting the existence of a suboptimal work distribution for every batching order can only be overcome by randomization. We omit the details due to reasons of space, but briefly explain how to randomize batching in the appendix, showing how to improve the expected speedup.

Finally, we conclude this section by comparing the new scheduler with an existing scheduler implementation used in the Scala Parallel Collections [17] in Figure 10. The Scala Parallel Collections scheduler is an example of an adaptive data-parallel scheduler relying a task-parallel scheduler under the hood ([15]). The batching order is chosen so that the sizes increase exponentially. At any point, the largest batch (task) is eligible for stealing - after a steal, the batch is divided in the same batching order. Due to the overheads of preemptively creating batch tasks and scheduling them, Scala Parallel Collections use a bound on the minimum batch size.

In Figure 10 we evaluate the performance of Scala Parallel Collections against the new scheduler against two benchmark applications - triangular matrix multiplication and Mandelbrot set computation. Triangular matrix multiplication has a linearly in- 
creasing workload. Scala Parallel Collections scale as the number of processors increases on both the i7 and the UltraSPARC machine, although they are slower by a constant factor. However, in the Mandelbrot set benchmark where we render set in the part of the plane ranging from $(-2,-2)$ to $(32,32)$, they do not scale beyond $P=2$ on the i7, and only start scaling after $P=16$ on the UltraSPARC. The reason is that the computationally expensive elements around the coordinates $(0,0)$ end up in a single batch and work on them cannot be parallelized. The work-stealing tree offers a more lightweight form of work-stealing with smaller batches and better load balancing.

\section{Conclusion}

We presented a scheduling algorithm for data-parallel operations that fulfills the specified criteria. Based on the experiments, we draw the following conclusions:

1. Minimum batch size on modern architectures needed to efficiently parallelize the sequential baseline typically ranges from a few dozen to several hundred elements.

2. There is no need to make batches larger than some architecture-specific size MAXSTEP, which is independent of the problem size - in fact, the approach employed by guided self-scheduling and factoring can be detrimental.

3. Batching can and should occur in isolation - by having workers communicate only when they run out of work batching can be more fine-grained (Figure 6).

4. Certain workloads require single element batches, in which case batch size has to be modified dynamically. Exponentially increasing batch size from 1 up to MAXSTEP works well for different workloads (Figure 9).

5. When the dominant part of the workload is distributed across a range of elements smaller than MAXSTEP, the worst-case speedup can be 1 . Randomizing the batching order can improve the average speedup.

We hinted that the work-stealing tree serves as a reduction tree, and we show the details in the appendix. We give some theoretical background to the conclusions from the experiments in the appendix as well. In the paper, we focused on parallel loops, but arrays, hash tables and trees are also eligible for parallel traversal [3] [17] [18]. The range iterator state was encoded with a single integer, but the state of other data structure iterators, as well as batching and stealing, may be more complex. While the CAS-based implementation of tryAdvance and trysteal ensures lock-freedom, CAS instructions in those methods can be replaced with short critical sections for more complicated iterators - the work-stealing tree algorithm is potentially applicable to other data structures in a straightforward way.

\section{References}

1. Nimar S. Arora, Robert D. Blumofe, and C. Greg Plaxton. Thread scheduling for multiprogrammed multiprocessors. In Proceedings of the tenth annual ACM symposium on Parallel algorithms and architectures, SPAA '98, pages 119-129, New York, NY, USA, 1998. ACM.

2. Robert D. Blumofe, Christopher F. Joerg, Bradley C. Kuszmaul, Charles E. Leiserson, Keith H. Randall, and Yuli Zhou. Cilk: An efficient multithreaded runtime system. In Journal of Parallel and Distributed Computing, pages 207-216, 1995. 
3. Antal Buss, Harshvardhan, Ioannis Papadopoulos, Olga Pearce, Timmie Smith, Gabriel Tanase, Nathan Thomas, Xiabing Xu, Mauro Bianco, Nancy M. Amato, and Lawrence Rauchwerger. Stapl: standard template adaptive parallel library. In Proceedings of the 3rd Annual Haifa Experimental Systems Conference, SYSTOR '10, pages 14:1-14:10, New York, NY, USA, 2010. ACM.

4. Manuel M. T. Chakravarty, Roman Leshchinskiy, Simon Peyton Jones, Gabriele Keller, and Simon Marlow. Data parallel haskell: a status report. In Proceedings of the 2007 workshop on Declarative aspects of multicore programming, DAMP '07, pages 10-18, New York, NY, USA, 2007. ACM.

5. Craig Chambers, Ashish Raniwala, Frances Perry, Stephen Adams, Robert R. Henry, Robert Bradshaw, and Nathan Weizenbaum. Flumejava: easy, efficient data-parallel pipelines. In Proceedings of the 2010 ACM SIGPLAN conference on Programming language design and implementation, PLDI '10, pages 363-375, New York, NY, USA, 2010. ACM.

6. David Chase and Yossi Lev. Dynamic circular work-stealing deque. In Proceedings of the seventeenth annual ACM symposium on Parallelism in algorithms and architectures, SPAA '05, pages 21-28, New York, NY, USA, 2005. ACM.

7. Guojing Cong, Sreedhar B. Kodali, Sriram Krishnamoorthy, Doug Lea, Vijay A. Saraswat, and Tong Wen. Solving large, irregular graph problems using adaptive work-stealing. In ICPP, pages 536-545, 2008.

8. Matteo Frigo, Charles E. Leiserson, and Keith H. Randall. The implementation of the cilk-5 multithreaded language. In Proceedings of the ACM SIGPLAN 1998 conference on Programming language design and implementation, PLDI '98, pages 212-223, New York, NY, USA, 1998. ACM.

9. Maurice Herlihy and Nir Shavit. The Art of Multiprocessor Programming. Morgan Kaufmann Publishers Inc., San Francisco, CA, USA, 2008.

10. Susan Flynn Hummel, Edith Schonberg, and Lawrence E. Flynn. Factoring: a method for scheduling parallel loops. Commun. ACM, 35(8):90-101, August 1992.

11. Intel Software Network. Intel Cilk Plus. http://cilkplus.org/.

12. Joseph JáJá. An Introduction to Parallel Algorithms. Addison-Wesley, 1992.

13. C. Koelbel and P. Mehrotra. Compiling global name-space parallel loops for distributed execution. Parallel and Distributed Systems, IEEE Transactions on, 2(4):440 -451, oct 1991.

14. Clyde P. Kruskal and Alan Weiss. Allocating independent subtasks on parallel processors. IEEE Trans. Softw. Eng., 11(10):1001-1016, October 1985.

15. Doug Lea. A java fork/join framework. In Java Grande, pages 36-43, 2000.

16. C. D. Polychronopoulos and D. J. Kuck. Guided self-scheduling: A practical scheduling scheme for parallel supercomputers. IEEE Trans. Comput., 36(12):1425-1439, December 1987.

17. Aleksandar Prokopec, Phil Bagwell, Tiark Rompf, and Martin Odersky. A generic parallel collection framework. In Proceedings of the 17th international conference on Parallel processing - Volume Part II, Euro-Par'11, pages 136-147, Berlin, Heidelberg, 2011. SpringerVerlag.

18. James Reinders. Intel threading building blocks. O’Reilly \& Associates, Inc., Sebastopol, CA, USA, first edition, 2007.

19. Vivek Sarkar. Optimized unrolling of nested loops. In Proceedings of the 14th international conference on Supercomputing, ICS '00, pages 153-166, New York, NY, USA, 2000. ACM.

20. Olivier Tardieu, Haichuan Wang, and Haibo Lin. A work-stealing scheduler for x10's task parallelism with suspension. In Proceedings of the 17th ACM SIGPLAN symposium on Principles and Practice of Parallel Programming, PPoPP '12, pages 267-276, New York, NY, USA, 2012. ACM.

21. T. H. Tzen and L. M. Ni. Trapezoid self-scheduling: A practical scheduling scheme for parallel compilers. IEEE Trans. Parallel Distrib. Syst., 4(1):87-98, January 1993. 


\section{A Appendix}

We provide the appendix section to further explain some of the concepts mentioned in the main paper which did not fit there. The information here is provided for convenience and it should not be necessary to read this section, but doing so may give useful insight.

\section{A.1 Work-stealing reduction tree}

As mentioned, the work-stealing tree is a particularly effective data-structure for a reduce operation. Parallel reduce is useful in the context of many other operations, such as finding the first element with a given property, finding the greatest element with respect to some ordering, filtering elements with a given property or computing an aggregate of all the elements (e.g. a sum).

There are two reasons why the work-stealing tree is amenable to implementing reductions. First, it preserves the order in which the work is split between processors, which allows using non-commutative operators for the reduce (e.g. computing the resulting transformation from a series of affine transformations can be parallelized by multiplying a sequence of matrices - the order is in this case important). Second, the reduce can largely be performed in parallel, due to the structure of the tree.

The work-stealing tree reduce works similar to a software combining tree [9], but it can proceed in a lock-free manner after all the node owners have completed their work, as we describe next. The general idea is to save the aggregated result in each node and then push the result further up the tree. Note that we did not save the return value of the kernel method in line 50 in Figure 5 , making the scheduler applicable only to parallelizing for loops. Thus, we add a local variable sum and update it each time after calling kernel. Once the node ends up in a COMPLETED or EXPANDED state, we assign it the value of sum. Note that updating an invocation-specific shared variable instead would not only break the commutativity, but also lead to the same bottleneck as we saw before with fixed-size batching. We therefore add two new fields with atomic access to Node, namely lresult and result. We also add a new field parent to Ptr. We expand the set of abstract node states with two additional ones, namely PREPARED and PUSHED. The expanded state diagram is shown in Figure 11.

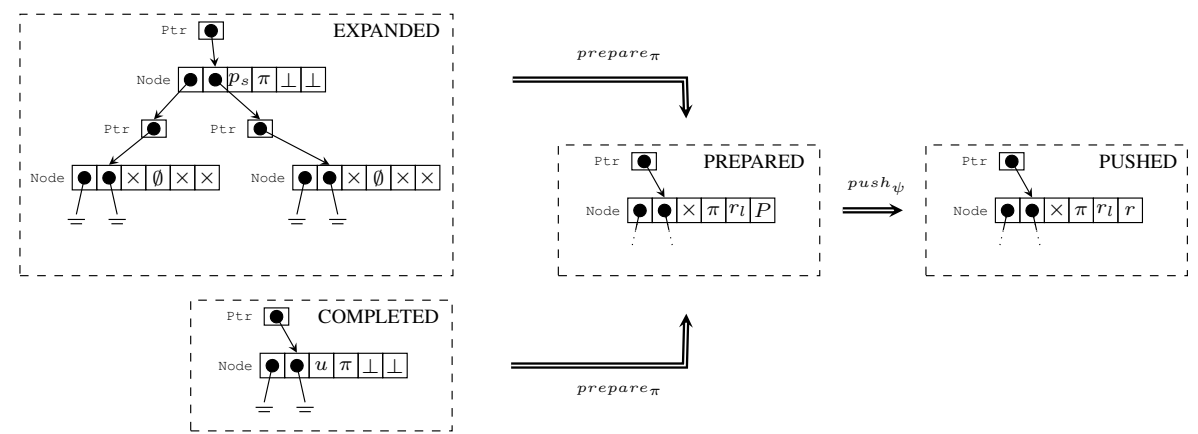

Fig. 11. Reduction state diagram

The parent field in Ptr is not shown in the diagram in Figure 11. The first two boxes in Node denote the left and the right child, respectively, as before. We represent the iteration state (progress) with a single box in Node. The iterator may either be stolen $\left(p_{s}\right)$ or completed $(u)$, but this is not important for the new states - we denote all such entries with $\times$. The fourth box represents the owner, the fifth and the sixth fields lresult and result. Once the work on the node is effectively completed, either due to a steal or a normal completion, the node owner 
the owner pushes the node into the PREPARED state in line 84, retrying in the case of spurious failures, and calls pushUp.

The method pushup may be invoked by the owner of the node attempting to write to the result field, or by another worker attempting to push the result up after having completed the work on one of the child nodes. The lresult field may not be yet assigned (line 92) if the owner has not completed the work - in this case the worker ceases to participate in the reduction and relies on the owner or another worker to continue pushing the result up. The same applies if the node is already in the PUSHED state (line 94). Otherwise, the lresult field can only be combined with the result values from the children if both children are in the PUSHED state. If the worker invoking pushUp notices that the children are not yet assigned the result, it will cease to participate in the reduction. Otherwise, it will compute the tentative result (line 104) and attempt to write it to result atomically with the CAS in line 106. A failed CAS triggers a retry, otherwise pushUp is called recursively on the parent node. If the current node is the root, the worker notifies any listeners that the final result is ready and the operations ends.

\section{A.2 Work-stealing tree traversal strategies}

We showed experimentally that changing the traversal order when searching for work can have a considerable effect on the performance of the work-stealing tree scheduler. We described these strategies briefly how, but did not present a precise, detailed pseudocode. In this section we show different implementations of the findWork and descend methods that lead to different tree traversal orders when stealing.

Assign. In this strategy a worker with index $i$ invoking $\mathrm{f} i \mathrm{ndW}$ ork picks a left-to-right traversal order at some node at level $l$ if and only if its bit at position $l \bmod \left\lceil\log _{2} P\right\rceil$ is 1 , that is:

$$
\left(i>>\left(l \bmod \left\lceil\log _{2} P\right\rceil\right)\right) \bmod 2=1
$$

The consequence of this is that when the workers descend in the tree the first time, they will pick different paths, leading to fewer steals assuming that the workload distribution is relatively uniform. If it is not uniform, then the workload itself should amortize the creation of extra nodes. We give the pseudocode in Figure 13.
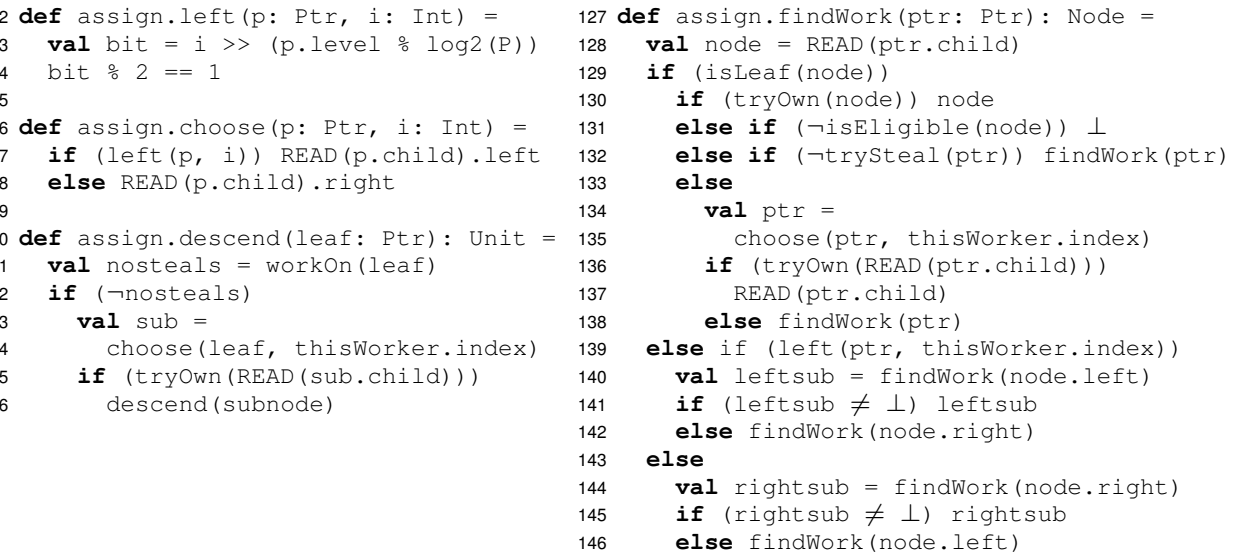

Fig. 13. Assign strategy 

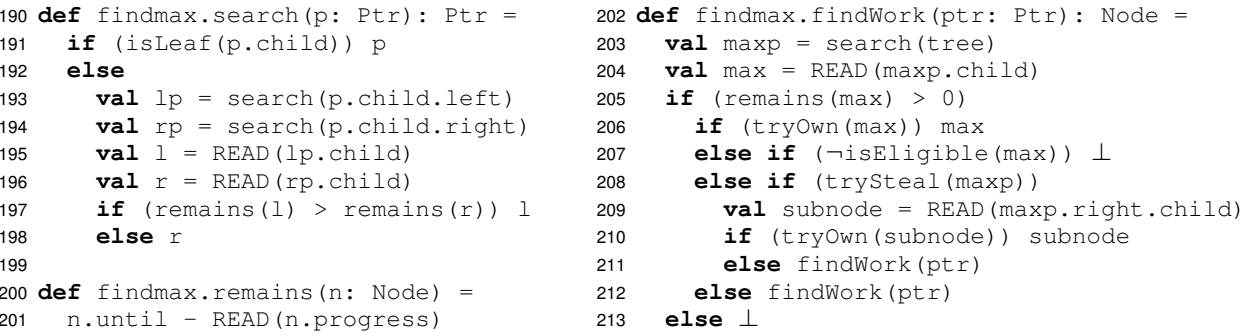

Fig. 16. FindMax strategy

\section{A.3 Speedup and optimality analysis}

In Figure 9-36 we identified a workload distribution for which the work-stealing reduction tree had a particularly bad performance. This coarse workload consisted of a major prefix of elements which required a very small amount of computation followed by a minority of elements which required a large amount of computation. We call it coarse because the number of elements was on the order of magnitude of a certain value we called MAXSTEP.

To recap, the speedup was suboptimal due to the following. First, to achieve an optimal speedup for at least the baseline, not all batches can have fewer elements than a certain number. We have established this number for a particular architecture and environment, calling it STEP. Second, to achieve an optimal speedup for ranges the size of which is below STEP.P, some of the batches have to be smaller than the others. The technique we apply starts with a batch consisting of a single element and increases the batch size exponentially up to MAXSTEP. Third, there is no hardware interrupt mechanism available to interrupt a worker which is processing a large batch, and software emulations which consist of checking a volatile variable within a loop are too slow when executing the baseline. Fourth, the worker does not know the workload distribution and cannot measure time. All this caused a single worker obtain the largest batch before the other workers had a chance to steal some work for a particular workload distribution. Justifying these claims requires a set of more formal definitions. We start by defining the context in which the scheduler executes.

Definition 1 (Oblivious conditions). If a data-parallel scheduler is unable to obtain information about the workload distribution, nor information about the amount of work it had previously executed, we say that the data-parallel scheduler works in oblivious conditions.

Assume that a worker decides on some batching schedule $c_{1}, c_{2}, \ldots, c_{k}$ where $c_{j}$ is the size of the $j$-th batch and $\sum_{j=1}^{k} c_{j}=N$, where $N$ is the size of the range. No batch is empty, i.e. $c_{j} \neq 0$ for all $j$. In oblivious conditions the worker does not know if the workload resembles the baseline mentioned earlier, so it must assume that it does and minimize the scheduling overhead. The baseline is not only important from a theoretical perspective being one of the potentially worst-case workload distribution, but also from a practical one - in many problems parallel loops have a uniform workload. We now define what this baseline means more formally.

Definition 2 (The baseline constraint). Let the workload distribution be a function $w(i)$ which gives the amount of computation needed for range element $i$. We say that a data-parallel scheduler respects the baseline constraint if and only if the speedup $s_{p}$ with respect to a sequential loop is arbitrarily close to linear when executing the workload distribution $w(i)=w_{0}$, where $w_{0}$ is the minimum amount of work needed to execute a loop iteration. 
Arbitrarily close here means that $\epsilon$ in $s_{p}=\frac{P}{1+\epsilon}$ can be made arbitrarily small.

The baseline constraint tells us that it may be necessary to divide the elements of the loop into batches, depending on the scheduling (that is, communication) costs. As we have seen in the experiments, while we should be able to make the $\epsilon$ value arbitrarily small, in practice it is small enough when the scheduling overhead is no longer observable in the measurement. Also, we have shown experimentally that the average batch size should be bigger than some value in oblivious conditions, but we have used particular scheduler instances. Does this hold in general, for every data-parallel scheduler? The answer is yes, as we show in the following lemma.

Lemma 1. If a data-parallel scheduler that works in oblivious conditions respects the baseline constraint then the batching schedule $c_{1}, c_{2}, \ldots, c_{k}$ is such that:

$$
\frac{\sum_{j=1}^{k} c_{j}}{k} \geq S(\epsilon)
$$

Proof. The lemma claims that in oblivious conditions the average batch size must be above some value which depends on the previously defined $\epsilon$, otherwise the scheduler will not respect the baseline constraint.

The baseline constraint states that $s_{p}=\frac{P}{1+\epsilon}$, where the speedup $s_{p}$ is defined as $T_{0} / T_{p}$, where $T_{0}$ is the running time of a sequential loop and $T_{p}$ is the running time of the scheduler using $P$ processors. Furthermore, $T_{0}=T \cdot P$ where $T$ is the optimal parallel running time for $P$ processors, so it follows that $\epsilon \cdot T=T_{p}-T$. We can also write this as $\epsilon \cdot W=W_{p}-W$. This is due to the running time being proportionate to the total amount of executed work, whether scheduling or useful work. The difference $W_{p}-W$ is exactly the scheduling work $W_{s}$, so the baseline constraint translates into the following inequality:

$$
W_{s} \leq \epsilon \cdot W
$$

In other words, the scheduling work has to be some fraction of the useful work. Assuming that there is a constant amount of scheduling work $W_{c}$ per every batch, we have $W_{s}=k \cdot W_{c}$. Lets denote the average work per element with $\bar{w}$. We then have $W=N \cdot \bar{w}$. Combining these relations we get $N \geq k \cdot \frac{W_{c}}{\epsilon \cdot \bar{w}}$, or shorter $N \geq k \cdot S(\epsilon)$. Since $N$ is equal to the sum of all batch sizes, we derive the following constraint:

$$
\frac{\sum_{j=1}^{k} c_{j}}{k} \geq \frac{W_{c}}{\epsilon \cdot \bar{w}}
$$

In other words, the average batch size must be greater than some value $S(\epsilon)$ which depends on how close we want to get to the optimal speedup. Note that this value is inversely proportionate to the average amount of work per element $\bar{w}-$ the scheduler could decide more about the batch sizes if it knew something about the average workload, and grows with the scheduling cost per batch $W_{c}$ - this is why it is especially important to make the workon method efficient. We already saw the inverse proportionality with $\epsilon$ in Figure 6. In part, this is why we had to make MAXSTEP larger than the chosen STEP (we also had to increase it due to increasing the scheduling work in workOn, namely, $W_{c}$ ). This is an additional constraint when choosing the batching schedule.

With this additional constraint there always exists a workload distribution for a given batching schedule such that the speedup is suboptimal, as we show next.

Lemma 2. Assume that $S(\epsilon)>1$, for the desired $\epsilon$. For any fixed batching schedule $c_{1}, c_{2}, \ldots, c_{k}$ there exists a workload distribution such that the scheduler executing it in oblivious conditions yields a suboptimal schedule. 
Proof. First, assume that the scheduler does not respect the baseline constraint. The baseline workload then yields a suboptimal speedup and the statement is trivially true because $S(\epsilon)>1$.

Otherwise, assume without the loss of generality that at some point in time a particular worker $\omega$ is processing some batch $c_{m}$ the size of which is greater or equal to the size of the other batches. This means the size of $c_{m}$ is greater than 1 , from the assumption. Then we can choose a workload distribution such that the work $W_{m}=\sum_{i=N_{m}}^{N_{m}+c_{m}} w(i)$ needed to complete batch $c_{m}$ is arbitrarily large, where $N_{m}=\sum_{j=1}^{m-1} c_{j}$ is the number of elements in the batching schedule coming before the batch $c_{m}$. For all the other elements we set $w(i)$ to be some minimum value $w_{0}$. We claim that the obtained speedup is suboptimal. There is at least one different batching schedule with a better speedup, and that is the schedule in which instead of batch $c_{m}$ there are two batches $c_{m_{1}}$ and $c_{m_{2}}$ such that $c_{m_{1}}$ consists of all the elements of $c_{m}$ except the last one and $c_{m_{2}}$ contains the last element. In this batching schedule some other worker can work on $c_{m_{2}}$ while $\omega$ works on $c_{m_{1}}$. Hence, there exists a different batching schedule which leads to a better speedup, so the initial batching schedule is not optimal.

We can ask ourselves what is the necessary condition for the speedup to be suboptimal. We mentioned that the range size has to be on the same order of magnitude as $S$ above, but can we make this more precise? We could simplify this question by asking what is the necessary condition for the worst-case speedup of 1 or less. Alas, we cannot find necessary conditions for all schedulers because they do not exist - there are schedulers which do not need any preconditions in order to consistently produce such a speedup (think of a sequential loop or, worse, a "scheduler" that executes an infinite loop). Also, we already saw that a suboptimal speedup may be due to a particularly bad workload distribution, so maybe we should consider only particular distributions, or have some conditions on them. What we will be able to express are the necessary conditions on the range size for the the existence of a scheduler which achieves a speedup greater than 1 on any workload. Since the range size is the only information known to the scheduler in advance, it can be used to affect its decisions in a particular implementation.

The worst-case speedups we saw occurred in scenarios where one worker (usually the invoker) started to work before all the other workers. To be able to express the desired conditions, we model this delay with a value $T_{d}$.

Lemma 3. Assume a data-parallel scheduler that respects the baseline constraint in oblivious conditions. There exists some minimum range size $N_{1}$ for which the scheduler can yield a speedup greater than 1 for any workload distribution.

Proof. We first note that there is always a scheduler that can achieve the speedup 1, which is merely a sequential loop. We then consider the case when the scheduler is parallelizing the baseline workload. Assume now that there is no minimum range size $N_{1}$ for which the claim is true. Then for any range size $N$ we must be able to find a range size $N+K$ such that the scheduler still cannot yield speedup 1 or less, for a chosen $K$. We choose $N=\frac{f \cdot T_{d}}{w_{0}}$, where $w_{0}$ is the amount of work associated with each element in the baseline distribution and $f$ is an architecture-specific constant describing the computation speed. The chosen $N$ is the number of elements that can be processed during the worker wakeup delay $T_{d}$. The workers that wake up after the first worker $\omega$ processes $N$ elements have no more work to do, so the speedup is 1 . However, for range size $N+K$ there are $K$ elements left that have not been processed. These $K$ elements could have been in the last batch of $\omega$. The last batch in the batching schedule chosen by the scheduler may include the $N$ th element. Note that the only constraint on the batch size is the lower bound value $S(\epsilon)$ from Lemma 1. So, if we choose $K=2 S(\epsilon)$ then either the last batch is smaller than $K$ or is greater than $K$. If it is smaller, then a worker different than $\omega$ will obtain and process the last batch, hence the speedup will be greater than 1 . If it is greater, then the worker $\omega$ will process the 
last batch - the other workers that wake up will not be able to obtain the elements from that batch. In that case there exists a better batching order which still respects the baseline constraint and that is to divide the last batch into two equal parts, allowing the other workers to obtain some work and yielding a speedup greater than 1 . This contradicts the assumption that there is no minimum range size $N_{1}$ - we know that $N_{1}$ is such that:

$$
\frac{f \cdot T_{d}}{w_{0}} \leq N_{1} \leq \frac{f \cdot T_{d}}{w_{0}}+2 \cdot S(\epsilon)
$$

Now, assume that the workload $w(i)$ is not the baseline workload $w_{0}$. For any workload we know that $w(i) \geq w_{0}$ for every $i$. The batching order for a single worker has to be exactly the same as before due to oblivious conditions. As a result the running time for the first worker $\omega$ until it reaches the $N$ th element can only be larger than that of the baseline. This means that the other workers will wake up by the time $\omega$ reaches the $N$ th element, and obtain work. Thus, the speedup can be greater than 1 , as before.

We have so far shown that we can decide on the average batch size if we know something about the workload, namely, the average computational cost of an element. We have also shown when we can expect the worst case speedup, potentially allowing us to take prevention measures. Finally, we have shown that any data-parallel scheduler deciding on a fixed schedule in oblivious conditions can yield a suboptimal speedup. Note the wording "fixed" here. It means that the scheduler must make a definite decision about the batching order without any knowledge about the workload, and must make the same decision every time - it must be deterministic. As hinted before, the way to overcome an adversary that is repetitively picking the worst case workload is to use randomization when producing the batching schedule. This is the topic of the next section.

\section{A.4 Overcoming the worst-case speedup using randomization}

Recall that the workload distribution that led to a bad speedup in our evaluation consisted of a sequence of very cheap elements followed by a minority of elements which were computationally very expensive. On the other hand, when we inverted the order of elements, the speedup became linear. The exponential backoff approach is designed to start with smaller batches first in hopes of hitting the part of the workload which contains most work as early as possible. This allow other workers to steal larger pieces of the remaining work, hence allowing a more fine grained batch subdivision. In this way the scheduling algorithm is workload-driven - it gives itself its own feedback. In the absence of other information about the workload, the knowledge that some worker is processing some part of the workload long enough that it can be stolen from is the best sign that the workload is different than the baseline, and that the batch subdivision can circumvent the baseline constraint. This heuristic worked in the example from figure 9-36 when the expensive elements were reached first, but failed when they were reached in the last, largest batch, and we know that there has to be a largest batch by Lemma 1 - a single worker must divide the range into batches the mean size of which has a lower bound. In fact, no other deterministic scheduler can yield an optimal speedup for all schedules, as shown by Lemma 2. For this reason we look into randomized schedulers.

In particular, in the example from the evaluation we would like the scheduler to put the smallest batches at the end of the range, but we have no way of knowing if the most expensive elements are positioned somewhere else. With this in mind we randomize the batching order. The baseline constraint still applies in oblivious conditions, so we have to pick different batch sizes with respect to the constraints from Lemma 1. Lets pick exactly the same set of exponentially increasing batches, but place consequent elements into different batches randomly. In other words, we permute the elements of the range and then apply the previous scheme. We expect some of 
the more expensive elements to be assigned to the smaller batches, giving other workers a higher opportunity to steal a part of the work.

In evaluating the effectiveness of this randomized approach we will assume a particular distribution we found troublesome. We define it more formally.

Definition 3 (Step workload distribution). A step workload distribution is a function which assigns a computational cost $w(i)$ to each element $i$ of the range of size $N$ as follows:

$$
w(i)= \begin{cases}w_{e}, & i \in\left[i_{1}, i_{2}\right] \\ w_{0}, & i \notin\left[i_{1}, i_{2}\right]\end{cases}
$$

where $\left[i_{1}, i_{2}\right]$ is a subsequence of the range, $w_{0}$ is the minimum cost of computation per element and $w_{e} \gg w_{0}$. If $w_{e} \geq f \cdot T_{d}$, where $f$ is the computation speed and $T_{d}$ is the worker delay, then we additionally call the workload highly irregular. We call $D=2^{d}=i_{2}-i_{1}$ the span of the step distribution. If $(N-D) \cdot \frac{w_{0}}{f} \leq T_{d}$ we also call the workload short.

We can now state the following lemma. We will refer to the randomized batching schedule we have described before as the randomized permutation with an exponential backoff. Note that we implicitly assume that the worker delay $T_{d}$ is significantly greater than the time $T_{c}$ spent scheduling a single batch (this was certainly true in our experimental evaluation).

Lemma 4. When parallelizing a workload with a highly irregular short step workload distribution the expected speedup inverse of a scheduler using randomized permutations with an exponential backoff is:

$$
\left\langle s_{p}^{-1}\right\rangle=\frac{1}{P}+\left(1-\frac{1}{P}\right) \cdot \frac{\left(2^{k}-2^{d}-1\right) !}{\left(2^{k}-1\right) !} \cdot \sum_{i=0}^{k-1} 2^{i} \frac{\left(2^{k}-2^{i}-1\right) !}{\left(2^{k}-2^{i}-2^{d}\right) !}
$$

where $D=2^{d} \gg P$ is the span of the step workload distribution.

Proof. The speedup $s_{p}$ is defined as $s_{p}=\frac{T_{0}}{T_{p}}$ where $T_{0}$ is the running time of the optimal sequential execution and $T_{p}$ is the running time of the parallelized execution. We implicitly assume that all processors have the same the same computation speed $f$. Since $w_{e} \gg w_{0}$, the total amount of work that a sequential loop executes is arbitrarily close to $D \cdot w_{e}$, so $T_{0}=\frac{D}{f}$. When we analyze the parallel execution, we will also ignore the work $w_{0}$. We will call the elements with cost $w_{e}$ expensive.

We assumed that the workload distribution is highly irregular. This means that if the first worker $\omega$ starts the work on an element from $\left[i_{1}, i_{2}\right]$ at some time $t_{0}$ then at the time $t_{1}=t_{0}+\frac{w_{e}}{f}$ some other worker must have already started working as well, because $t_{1}-t_{0} \geq T_{d}$. Also, we have assumed that the workload distribution is short. This means that the first worker $\omega$ can complete work on all the elements outside the interval $\left[i_{1}, i_{2}\right]$ before another worker arrives. Combining these observations, as soon as the first worker arrives at an expensive element, it is possible for the other workers to parallelize the rest of the work.

We assume that after the other workers arrive there are enough elements left to efficiently parallelize work on them. In fact, at this point the scheduler will typically change the initially decided batching schedule - additionally arriving workers will steal and induce a more finegrained subdivision. Note, however, that the other workers cannot subdivide the batch on which the current worker is currently working on - that one is no longer available to them. The only batches with elements of cost $w_{e}$ that they can still subdivide are the ones coming after the first batch in which the first worker $\omega$ found an expensive element. We denote this batch with $c_{\omega}$. The batch $c_{\omega}$ may, however, contain additional expensive elements and the bigger the batch the more 
probable this is. We will say that the total number of expensive elements in $c_{\omega}$ is $X$. Finally, note that we assumed that $D \gg P$, so our expression will only be an approximation if $D$ is very close to $P$.

We thus arrive at the following expression for speedup:

$$
s_{p}=\frac{D}{X+\frac{D-X}{P}}
$$

Speedup depends on the value $X$. But since the initial batching schedule is random, the speedup depends on the random variable and is itself random. For this reason we will look for its expected value. We start by finding the expectation of the random variable $X$.

We will now solve a more general problem of placing balls to an ordered set of bins and apply the solution to finding the expectation of $X$. There are $k$ bins, numbered from 0 to $k-1$. Let $c_{i}$ denote the number of balls that fit into the $i$ th bin. We randomly assign $D$ balls to bins, so that the number of balls in each bin $i$ is less than or equal to $c_{i}$. In other words, we randomly select $D$ slots from all the $N=\sum_{i=0}^{k-1} c_{i}$ slots in all the bins together. We then define the random variable $X$ to be the number of balls in the non-empty bin with the smallest index $i$. The formulated problem corresponds to the previous one - the balls are the expensive elements and the bins are the batches.

An alternative way to define $X$ is as follows:

$$
X=\sum_{i=0}^{k-1} \begin{cases}\text { number of balls in bin } i & \text { if all the bins } j<i \text { are empty } \\ 0 & \text { otherwise }\end{cases}
$$

Applying the linearity property, the expectation $\langle X\rangle$ is then:

$$
\langle X\rangle=\sum_{i=0}^{k-1}\langle\text { number of balls in bin } i \text { given that all the bins } j<i \text { are empty, and } 0 \text { otherwise }\rangle
$$

The expectation in the sum is conditional on the event that all the bins coming before $i$ are empty. We call the probability of this event $p_{i}$. We define $b_{i}$ as the number of balls in any bin $i$. From the properties of conditional expectation we than have:

$$
\langle X\rangle=\sum_{i=0}^{k-1} p_{i} \cdot\left\langle b_{i}\right\rangle
$$

The number of balls in any bin is the sum of the balls in all the slots of that bin which spans slots $n_{i-1}$ through $n_{i-1}+c_{i}$. The expected number of balls in a bin $i$ is thus:

$$
\left\langle b_{i}\right\rangle=\sum_{i=n_{i-1}}^{n_{i-1}+c_{i}}\langle\text { expected number of balls in a single slot }\rangle
$$

We denote the total capacity of all the bins $j \geq i$ as $q_{i}$ (so that $q_{0}=N$ and $q_{k-1}=2^{k-1}$ ). We assign balls to slots randomly with a uniform distribution - each slot has a probability $\frac{D}{q_{i}}$ of being selected. Note that the denominator is not $N$ - we are calculating a conditional probability for which all the slots before the $i$ th bin are empty. The expected number of balls in a single slot is thus $\frac{D}{q_{i}}$. It follows that:

$$
\left\langle b_{i}\right\rangle=c_{i} \cdot \frac{D}{q_{i}}
$$


Next, we compute the probability $p_{i}$ that all the bins before the bin $i$ are empty. We do this by counting the events in which this is true, namely, the number of ways to assign balls in bins $j \geq i$. We will pick combinations of $D$ slots, one for each ball, from a set of $q_{i}$ slots. We do the same to enumerate all the assignments of balls to bins, but with $N=q_{0}$ slots, and obtain:

$$
p_{i}=\frac{\left(\begin{array}{l}
q_{i} \\
D
\end{array}\right)}{\left(\begin{array}{c}
q_{0} \\
D
\end{array}\right)}
$$

We assumed here that $q_{i} \geq D$, otherwise we cannot fill all $D$ balls into bins. We could create a constraint that the last batch is always larger than the number of balls. Instead, we simply define $\left(\begin{array}{c}q_{i} \\ D\end{array}\right)=0$ if $q_{i}<D$ - there is no chance we can fit more than $q_{i}$ balls to $q_{i}$ slots. Combining these relations, we get the following expression for $\langle X\rangle$ :

$$
\langle X\rangle=D \cdot \frac{\left(q_{0}-D\right) !}{q_{0} !} \sum_{i=0}^{k-1} c_{i} \cdot \frac{\left(q_{i}-1\right) !}{\left(q_{i}-D\right) !}
$$

We use this expression to compute the expected speedup inverse. By the linearity of expectation:

$$
\left\langle s_{p}^{-1}\right\rangle=\frac{1}{P}+\left(1-\frac{1}{P}\right) \cdot \frac{\left(q_{0}-D\right) !}{q_{0} !} \sum_{i=0}^{k-1} c_{i} \cdot \frac{\left(q_{i}-1\right) !}{\left(q_{i}-D\right) !}
$$

This is a more general expression than the one in the claim. When we plug in the exponential backoff batching schedule, i.e. $c_{i}=2^{i}$ and $q_{i}=2^{k}-2^{i}$, the lemma follows.

The expression derived for the inverse speedup does not have a neat analytical form, but we can evaluate it for different values of $d$ to obtain a diagram. As a sanity check, the worst expected speedup comes with $d=0$. If there is only a single expensive element in the range, then there is no way to parallelize execution - the expression gives us the speedup 1 . We expect a better speedup as $d$ grows - when there are more expensive elements, it is easier for the scheduler to stumble upon some of them. In fact, for $d=k$, with the conventions established in the proof, we get that the speedup is $\frac{1}{P}+\left(1-\frac{1}{P}\right) \cdot \frac{c_{0}}{D}$. This means that when all the elements are expensive the proximity to the optimal speedup depends on the size $c_{0}$ of the first batch - the less elements in it, the better. Together with the fact that many applications have uniform workloads, this is also the reason why we advocate exponential backoff for which the size of the first batch is 1 .

We call the term $\frac{\left(q_{0}-D\right) !}{q_{0} !} \sum_{i=0}^{k-1} c_{i} \cdot \frac{\left(q_{i}-1\right) !}{\left(q_{i}-D\right) !}$ the slowdown and plot it with respect to span $D$ on the diagram in Figure 17. In this diagram we choose $k=10$, and the number of elements $N=$ $2^{10}=1024$. As the term nears 1 , the speedup nears 1 . As the term approaches 0 , the speedup approaches the optimal speedup $P$. The quicker the term approaches 0 as we increase $d$, the better the scheduler. We can see that fixed-size batching should work better than the exponential backoff if the span $D$ is below 10 elements, but is much worse than the exponential backoff otherwise. Linearly increasing the batch size from 0 in some step $a=\frac{2 \cdot\left(2^{k}-1\right)}{k \cdot(k-1)}$ seems to work well even for span $D<10$. However, the mean batch size $\overline{c_{i}}=\frac{S}{k}$ means that this approach may easily violate the baseline constraint, and for $P \approx D$ the formula is an approximation anyway.

The conclusion is that selecting a random permutation of the elements should work very well in theory. For example, the average speedup becomes very close to optimal if less than $D=10$ elements out of $N=1024$ are expensive. However, randomly permuting elements would in practice either require a preparatory pass in which the elements are randomly copied or would require the workers to randomly jump through the array, leading to cache miss issues. In both cases the baseline performance would be violated. Even permuting the order of the batches seems problematic, as it would require storing information about where each batch started and 


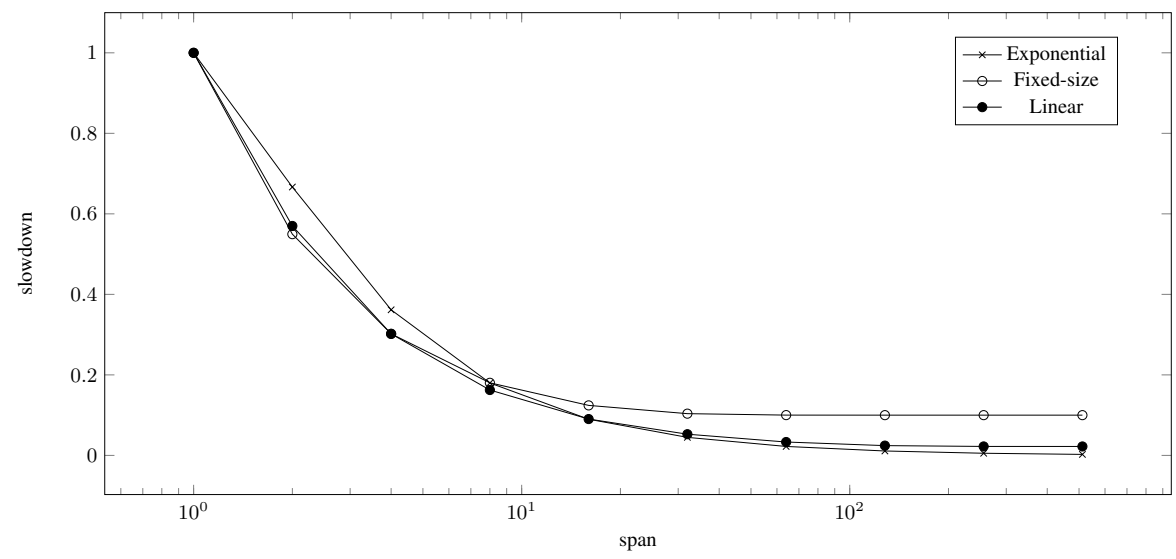

Fig. 17. Randomized scheduler executing step workload - speedup vs. span

left off, as well as its intermediate result - for something like that we need a data structure like a work-stealing tree and we saw that we have to minimize the number of nodes there as much as possible.

There are many approaches we could study, many of which could have viable implementations, but we focus on a particular one which seems easy to implement for ranges and other data structures. Recall that in the example in Figure 9-36 the interval with expensive elements was positioned at the end of the range. What if the worker alternated the batch in each step by tossing the coin to decide if the next batch should be from the left (start) or from the right (end)? Then the worker could arrive at the expensive interval on the end while the batch size is still small with a relatively high probability. The changes to the work-stealing tree algorithm are minimal - in addition to another field called rresult (the name of which should shed some light on the previous choice of name for lresult), we have to modify the workOn, complete and pushUp methods. While the latter two are straightforward, the lines 47 through 51 of work on are modified. The new work On method is shown in Figure 18.

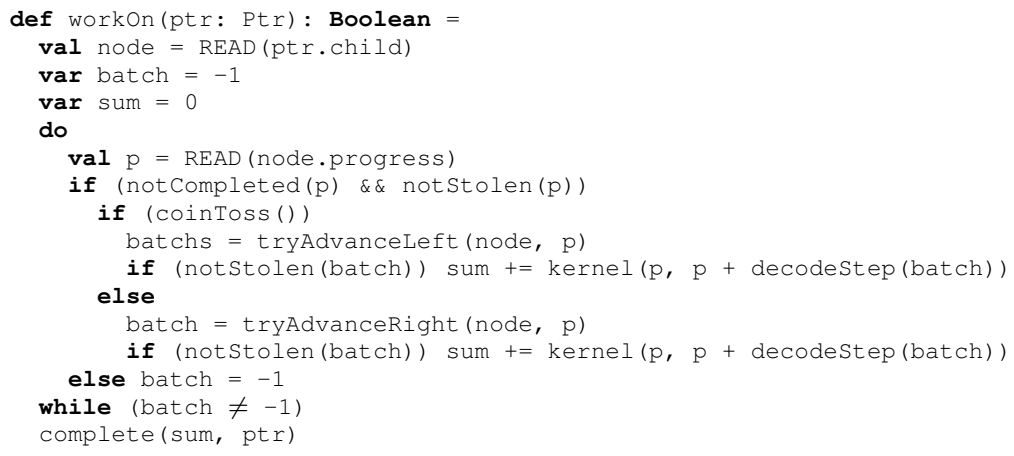

Fig. 18. Randomized loop method 
The main issue here is to encode and atomically update the iteration state, since it consists of two pieces of information - the left and the right position in the subrange. We can encode these two positions by using a long integer field and a long CAS operation to update it. The initial 32 bits can contain the position on the left side of the subrange and the subsequent 32 on the right side. With this in mind, the methods tryAdvanceleft, tryAdvanceRight, not Stolen, not Completed and decodeStep should be straightforward.
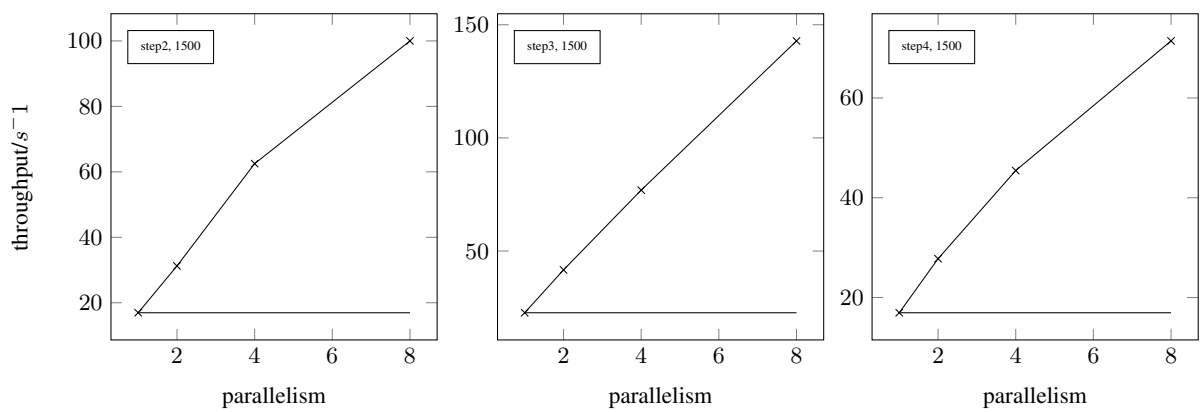

Fig. 19. The randomized work-stealing tree and the STEP3 workload

We evaluate the new scheduler on the distribution from Figure 9-36 and show the results in Figure 19. The first two diagrams (STEP2 and STEP3) show that with the expensive interval at the beginning and the end of the range the work-stealing tree achieves a close to optimal speedup. However, there is still a worst case scenario that we have to consider, and that is to have a step workload with the expensive interval exactly in the middle of the range. Intuition tells us that the probability to hit this interval early on is smaller, since a worker has to progress through more batches to arrive at it. The workload STEP4 in the third diagram of Figure 19 contains around $25 \%$ expensive elements positioned in the middle of the range. The speedup is decent, but not linear for STEP4, since the bigger batches seem to on average hit the middle of the range more often.

Having shown that randomization does help scheduling both in theory and in practice, we conclude that the problem of overcoming particularly bad workload distributions is an algorithmic problem of finding a batching schedule which can be computed and maintained relatively quickly, leaving this task as future work. 